\title{
POSSIBLE LONG-TERM IMPROVEMENTS TO THE ADVANCED PHOTON SOURCE *
}

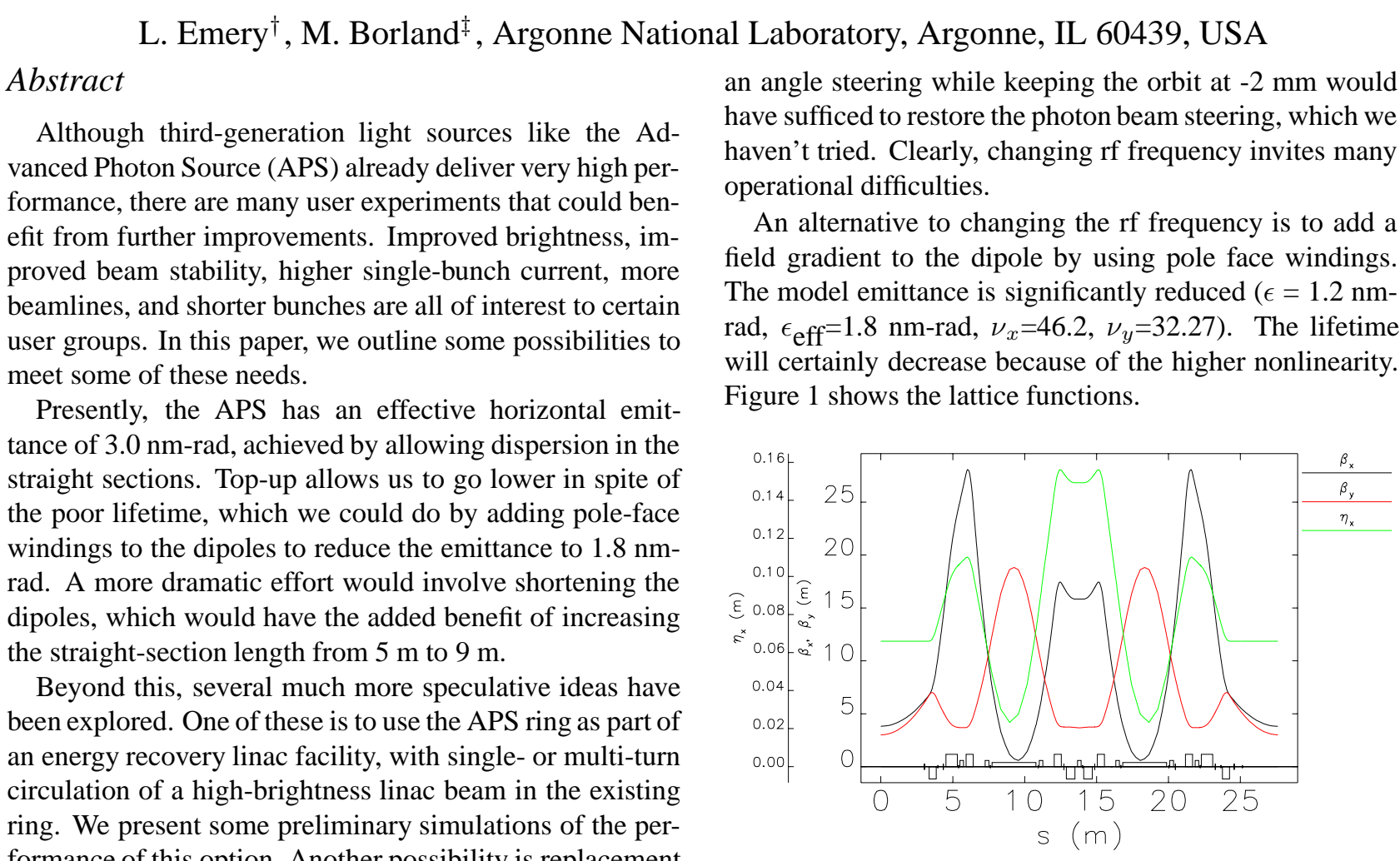
formance of this option. Another possibility is replacement of the APS storage ring with a next-generation ring capable of ultra-low emittance and making full use of top-up. We present a concept for a facility that promises 75 pm-rad horizontal emittance and $10 \mathrm{~m}$-long straight sections.

\section{INTRODUCTION}

Several ideas for improving the 7-GeV Advanced Photon Source (APS) are presented here. The main features and the identification of technological areas that needs further exploration are described.

\section{POSSIBLE MODIFICATIONS OF THE APS STORAGE RING}

The emittance can be reduced by increasing the horizontal damping partition number through a change in $\mathrm{rf}$ frequency. We have reduced the emittance during studies from $3.0 \mathrm{~nm}$-rad to $2.5 \mathrm{~nm}$-rad. While the rf frequency was changed in steps, the values of tunes and chromaticity were adjusted manually to keep them constant. Because of dispersion, the orbit at the straight section was steered horizontally by about $-2 \mathrm{~mm}$. When steering the beam back, the emittance returned close to the original value. Perhaps

\footnotetext{
* Work supported by U.S. Department of Energy, Office of Basic Energy Sciences under Contract No. W-31-109-ENG-38.

† emery@aps.anl.gov

† borland@aps.anl.gov
}

Figure 1: Machine functions of a much lower emittance lattice cell with gradients in the dipole.

The normalized gradient $k$ for the dipole is optimized at $-0.17 \mathrm{~m}^{-2}$. The field in the dipole gap will vary from $0.2 \mathrm{~T}$ to $1.0 \mathrm{~T}$, which is a large relative range. The pole winding could either cover the $10-\mathrm{cm}$ width of the pole or cover only about $+/-3 \mathrm{~cm}$ of good field region to reduce the range in field, though one should be mindful of fringe fields. The current sheet value is given by $I=$ $\left(k(B \rho) g_{1 / 2} w\right) /\left(4 \pi \times 10^{-7}\right)$. With $k=-0.17 \mathrm{~m}^{-2}, B \rho=$ $23.3 \mathrm{~T}-\mathrm{m}$, half-gap $g_{1 / 2}=0.03 \mathrm{~m}$, and width $w=0.105$ $\mathrm{m}$, the required $I$ is $10 \mathrm{kA}$-turn. Since a maximum of 10 $\mathrm{A} / \mathrm{mm}^{2}$ for cooled conductors might be possible according to [1], the coil thickness could be as small as $10 \mathrm{~mm}$. Cooling limits of conductors should be further explored to see if the coil thickness can be reduced.

The fact that all APS multipoles have individual power supplies allows gradual changes to the machine configuration, so we could implement such changes over a series of one-month shutdowns.

As previously reported in [2], lengthening the straight sections enables users to install three or possibly four undulators for greater flexibility of operation. This would not only increase the brightness for users who demand it, it would provide multiple beamlines from a single straight section for applications that are less demanding. Presently about $\mathrm{L}=5.0 \mathrm{~m}$ is available for installation of undula- 
tors in the 5.9-m straight section. Removing the nearest quadrupole magnet from the two adjacent girders, or replacing the dipoles with shorter ones, or both would allow space for an extra ID or two. One option is to replace the two closest $0.6-\mathrm{T}, 3-\mathrm{m}$-long dipoles with $1.8-\mathrm{T}, 1-\mathrm{m}$-long gradient dipoles. These special solutions have increased emittance, so we may only allow one or a few such straight section modifications. Various configurations are presently being pursued [3].

\section{ENERGY RECOVERY LINAC}

In addition to advanced storage rings and FELs, another concept for a next-generation light source is the Energy Recovery Linac (ERL) $[4,5]$. In this concept, a superconducting linac is used to accelerate high-quality electron bunches to multi-GeV energies. The bunches are passed through one or more undulators in the course of being bent around a race-track transport line, which brings them back to the head of the linac. If the path length is such as to delay the bunch phase by 180 degrees, then the energy is extracted from the beam, which is then dumped at a relatively low energy. In this way, a high-duty-factor beam can be provided with relatively modest energy consumption.

A variation on the ERL concept involves injecting the beam from a superconducting linac into a storage ring, where it is allowed to circulate one or more turns. This has two potential advantages: First, the bunch rate from the linac injector can potentially be reduced without reducing the average flux delivered to users. Second, if this is done with an existing storage ring, then existing undulators and beamlines potentially benefit from higher beam quality and shorter bunches. In such a scenario, beam would be extracted from the ring after circulating one more more turns and then be sent back into the linac in the opposite direction.

We performed a preliminary evaluation of the possibility of such a facility at the APS, using the existing APS storage ring. Of particular concern were incoherent and coherent synchrotron radiation (ISR and CSR) effects. We used elegant to investigate three lattices: a lattice with zerodispersion in the straight sections ("ZD" lattice), a lattice with low emittance ("LE" lattice), and an isochronous lattice with minimized emittance ("ISO" lattice). For each lattice, we injected a 50 -pC bunch with $0.01 \%$ initial rms energy spread, $1-\mu \mathrm{m}$-rad initial normalized rms emittance, and 50- $\mu \mathrm{m}$ initial rms bunch length. The ZD lattice was the clear winner in terms of emittance, with less than $10 \%$ emittance growth in the first turn. This is because energy spread due to CSR does not result in effective emittance growth in the straight sections. In the longitudinal plane, the lattices are fairly close. Figure 2 shows simulation results for a single turn.

It must be noted that these simulations used a Gaussian input beam, which may be very misleading [6]. A more trustworthy simulation requires a complete simulation of the injector, including bunch compression. Any such prob- lems can be mitigated by making the initial bunch longer and increasing the energy spread. Simply increasing the initial bunch length to $100 \mu \mathrm{m}$ allows one to increase the charge to $100 \mathrm{pC}$ while keeping the emittance growth under $10 \%$. In addition, using a longer bunch opens the option of circulating for many turns. Figure 3 shows simulation results for a 100-pC bunch with initial rms bunch length of $300 \mu \mathrm{m}$ (1 ps). The modest emittance growth suggests that a multiturn scenario would be feasible.

In addition to multiturn tracking and use of a realistic initial bunch distribution, these simulations should be extended to include wakefields. The recent development of an impedance database for the APS [7] will be useful in this regard.
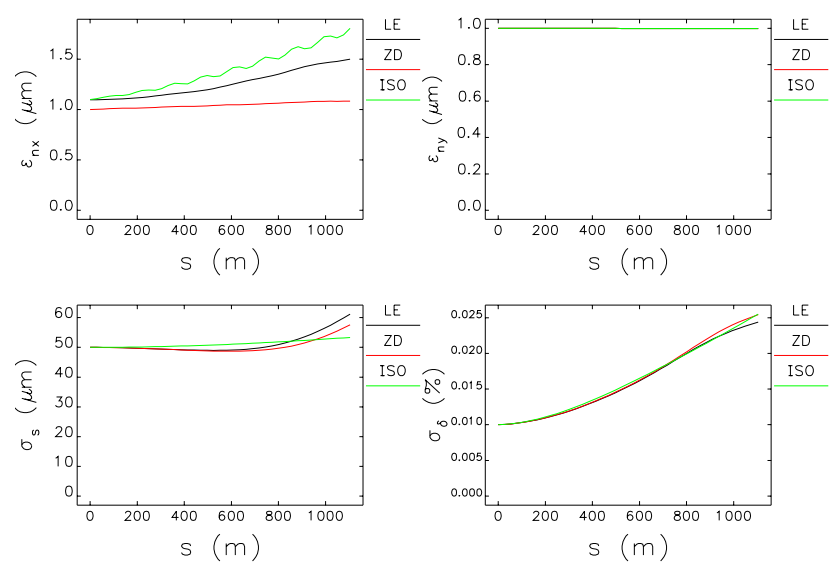

Figure 2: Normalized rms $\mathrm{x}$ and y emittances, rms bunch length, and rms momentum spread vs distance, for one turn in APS of a 50-pC bunch, for three trial lattices.
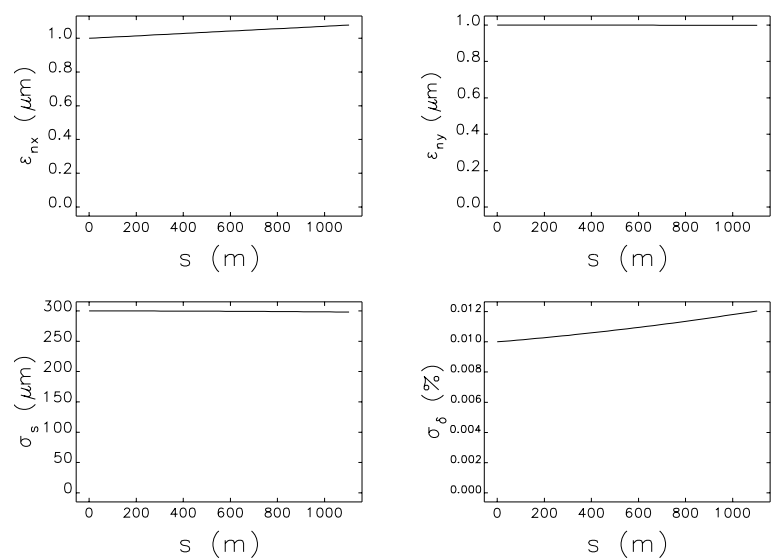

Figure 3: Normalized rms $\mathrm{x}$ and $\mathrm{y}$ emittances, rms bunch length, and rms momentum spread vs distance, for one turn in APS of a 100-pC bunch for the ZD lattice. The initial bunch length is $300 \mu \mathrm{m}$.

\section{REPLACEMENT STORAGE RINGS}

Another way to enhance the existing APS beamlines is to replace the APS ring with a new, lower-emittance ring. We investigated several possibilities for this. The most obvious 
improvement is to double the number of cells in the lattice, which should reduce the emittance eightfold. In addition to this, we have investigated a more extreme concept that could reduce the emittance 100 -fold.

For the 80-sector exercise, we started with the present APS design and then cut the length of all magnets and drift spaces in half, producing two subsectors in each present sector. We eliminated the straight section and triplets from the center, allowing us to lengthen the existing straight section to about $7.6 \mathrm{~m}$ from the present $4.8 \mathrm{~m}$. elegant was used to minimize the effective emittance subject to various contraints on the beta functions. Figure 4 shows the lattice functions for this concept, which has a nominal effective emittance of $0.3 \mathrm{~nm}$-rad.

The 80-sector ring is only about ten times better than the present-day APS, and may seem to offer too little improvement to justify the cost. To get truly low emittance, we developed a somewhat speculative concept called the eXtreme Photon Source (XPS). It features 40 sectors with three strong gradient dipoles per sector. Instead of separate quadrupoles and sextupoles, we assumed combinedfunction 12-pole magnets. Since there is dispersion everywhere, this promises to allow correction of the chromaticity from each quadrupole at the quadrupole itself. To improve stability and make the magnets feasible, we also suggest that all magnets be of a variable permanent magnet design. Figure 5 shows the lattice functions. The nominal effective emittance is 75 pm-rad, with a $20 \%$ increase expected from intrabeam scattering (assuming full coupling). The lattice allows approximately $10 \mathrm{~m}$ for insertion devices in each straight section.

Because of the strong sextupoles in this lattice, the dynamic aperture is too small to allow accumulation. In addition, the beam lifetime is estimated at about 45 minutes assuming $1 \mathrm{~mA} /$ bunch and 7.5 MV rf voltage. Hence, we would have to use on-axis injection and inject frequently, using a high-current, low-emittance injector. We propose using the existing APS ring (moved to the floor of the tunnel) as an accumulator ring for the XPS ring. In this proposal, both the APS and XPS would have approximately the same stored beam current at all times. When the beam in the XPS had decayed excessively, it would be swapped with the beam in the accumulator during a single turn. (This entails filling only half the circumference in each machine.) After swapping, the bunches in the former XPS beam would be replenished with the injector and then kept ready using top-up until the next "swap-up" was required. An advantage of using on-axis injection is that we can operate on the coupling resonance, thus providing round beams and smaller horizontal emitance.

The dynamic aperture for the bare lattice without errors is about $\pm 0.75 \mathrm{~mm}$ in both planes for energy offsets from $-2 \%$ to $2 \%$. Because of the small emittance, this corresponds to more than 20 times the rms beam size. Clearly, much more work is required on this concept, but it appears promising. Note that the normalized emittance of this 7$\mathrm{GeV}$ ring is about $0.5 \mu \mathrm{m}$, comparable to proposed ERL and FEL linacs. Like ERL and (to a lesser extent) FEL proposals, the XPS is highly speculative and requires new technology. Our studies indicate that investigation of the requirements for such a ring could be very worthwhile.

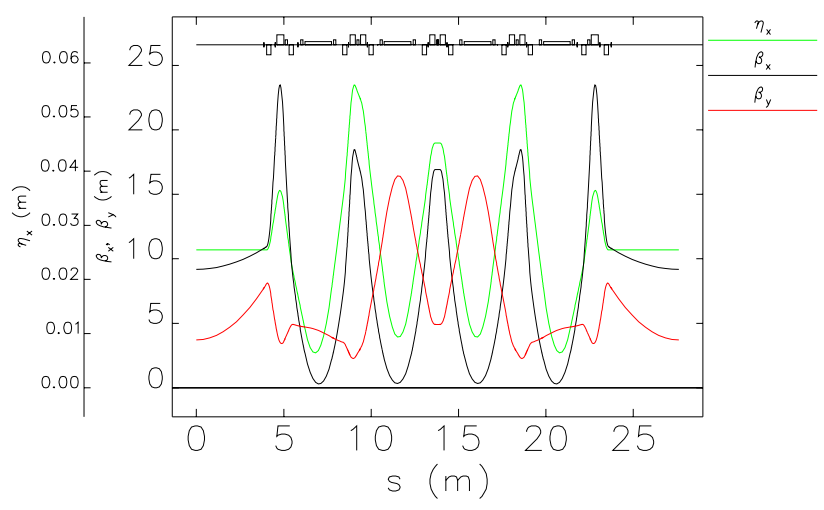

Figure 4: Lattice functions for the 80-sector concept replacement ring.

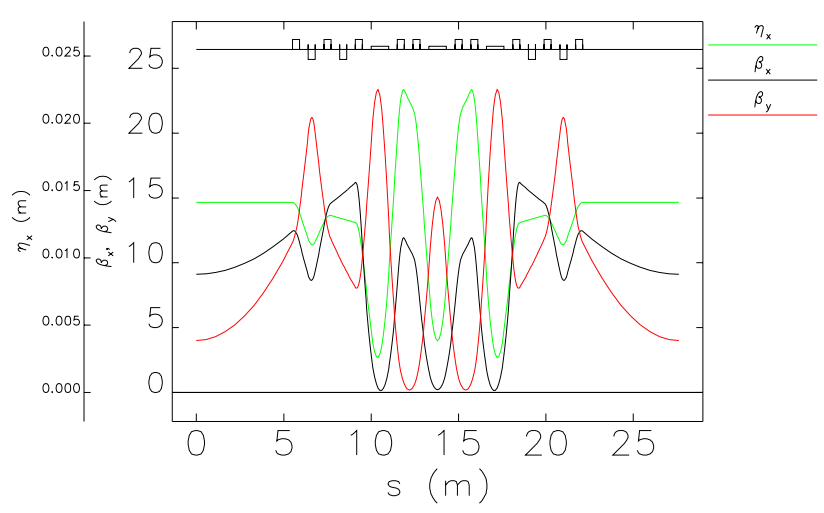

Figure 5: Lattice functions for the eXtreme Photon Source concept replacement ring.

\section{REFERENCES}

[1] G. E. Fischer, AIP Conference Proceedings 153, 1120 (1987).

[2] L. Emery et al. "Progress and Prospects Toward Brightness Improvements at the Advanced Photon Source," 2001 PAC, Chicago, 2602 (2002).

[3] V. Sajaev, private communication.

[4] S. Gruner, D. Bilderback, and M. Tigner, "Synchrotron Radiation Sources for the Future," white paper, http://erl.chess.cornell.edu/Papers/Papers.htm.

[5] I. Ben-Zvi et al., "Photoinjected Energy Recovery Linac Upgrade for the National Synchrotron Light Source," 2001 PAC, Chicago, 350 (2002).

[6] M. Borland et al., "Start-to-End Simulation of Self-Amplified Spontaneous Emission Free-Electron Lasers from the Gun through the Undulator," NIM A 483 (2002) 268-272.

[7] Y. Chae, "The Impedance Database and Its Application to the APS Storage Ring," these proceedings. 